\title{
Learning More from What We Already Know About Childhood Obesity Prevention
}

\author{
Shiriki K. Kumanyika, PhD, MPH
}

\section{Introduction}

$\mathbf{T}$

he persistent epidemic of population-wide obesity has put a spotlight on the need for greater understanding of what obesity research is telling us about effective long-term solutions. ${ }^{1}$ The number of reports and recommendations on what to do about the problem is impressive. For example, the Institute of Medicine Committee on Accelerating Progress in Obesity Prevention reviewed $>800$ reports to identify policy, systems, and environmental change strategies most well supported by evidence. ${ }^{2}$ There have been some signs of progress among the youngest children., ${ }^{3,4}$ However, progress is not yet stable or visible in the national trend data, and even where observed in states or localities, progress has not been translated into clearly scalable approaches. ${ }^{5}$ Moreover, there is well-founded concern about a lack of progress in reducing obesity prevalence in racial/ ethnic minority populations and in low-resource settings where disparities vis a vis reference populations are observed. ${ }^{6}$ Taken together, the lack of evidence of progress overall and especially with respect to health disparities has led to calls for new approaches. ${ }^{1,7-9}$

The Childhood Obesity Evidence Base (COEB) project, described in this supplement, is a novel approach for deriving new insights from existing evidence. The project demonstrates an approach for identifying and revisiting a set of studies for which separate findings are already known, by breaking down and reaggregating study specifics in a way that supports further analyses. COEB developed and piloted the use of a taxonomic approach to code and categorize elements of US obesity prevention studies, with a focus on children of ages 2 to 5 years, to show how this approach can facilitate understanding of which intervention strategies work and under what circumstances. COEB was undertaken under the auspices of the National Collaborative on Childhood Obesity Research as a next step in advancing the conceptual and methodological infrastructure of childhood obesity research. Four supplement articles provide details on the rationale and methods for the development of taxonomies, resulting database, rationale for taxonomic vs. conventional metaanalysis, and a meta-analysis example. ${ }^{10-13}$ After a brief description of the COEB project, this commentary reflects on the importance and potential implications of the project from a methodological perspective. Another commentary considers implications in the context of current thinking about scientific directions in the childhood obesity prevention field. ${ }^{14}$

\section{The Taxonomic Meta-Analysis Approach}

In brief, the COEB team conducted an inclusive scoping review of US childhood obesity prevention studies conducted between 2005 and 2019 with children in the targeted age range. The taxonomic approach was piloted using the subset of 51 of the 147 studies identified that reported a weight status outcome. The process involved detailed coding of reported study elements, related to outcomes, intervention components, intended recipients of the intervention, and aspects of the intervention contexts. The coded elements were then grouped into categories and subcategories based on conceptual similarities and relationships (Table 1). Results for each taxonomic component were tabulated according to frequency of occurrence and associated with one or more levels of intervention according to a socioecological model, that is, individual, interpersonal, organizational, community, and societal level. ${ }^{15}$ Thus, the project has so far contributed to the evidence base on early childhood obesity prevention interventions in three ways: (1) results of a scoping review of studies conducted with 2- to 5 -year-old children during the past 15 years, (2) a database of these studies coded according to an empirically derived classification of key study variables (i.e., not limited to categories based on existing theory), and (3) an example of one way to use the database in meta-analysis. The database can be used to support a variety of analysis types and questions. 
Table I. Categories and Subcategories for Each of the Taxonomies Developed in the COEB Project for Four Outcomes: Weight Status, Physical Activity, Diet, and Sleep

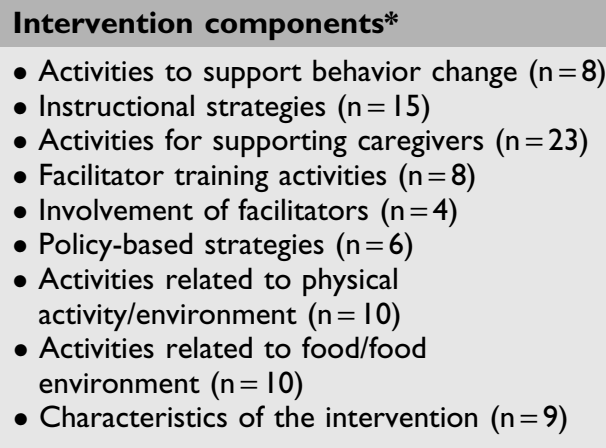

Intended recipients

- Level of education

- Age group

- Living arrangements

- Gender

- Language spoken at home

- Physical/learning differences

- Race/ethnicity

- Socioeconomic status

- Technology access

- Health status
Intervention context

- Community type

- Geographic location

- Intervention setting

- Instructor/facilitator education and experience

- Instructor/facilitator gender

- Instructor/facilitator language

- Instructor/facilitator race/ethnicity

- School grade level

- School/district/community language status

- School/district/community race/ethnicity composition

- School/district/community socioeconomic status

- Caregiver/parent employment status

- Caregiver/parent health status

- Caregiver/parent relationship status

- Caregiver/parent language status

- Caregiver/parent age

- Technology present in home

Adapted from King et al. ${ }^{10}$

${ }^{a} n=$ number of subcategories.

\section{Research Translation for Improved Outcomes and Diverse Contexts}

The COEB project comes at a good time. We have a wealth of information about what to do but recognize the need to be more systematic and granular in understanding how to transfer or translate this knowledge for effectiveness in diverse natural settings. Systematic scientific approaches to such translation involve implementation research: developing informed hypotheses about variations in interventions, intended recipients, and implementation settings that can influence effectiveness and studying ways to address these influences. Taxonomic approaches can be useful in evaluating existing interventions and developing new hypotheses for testing.

Increased awareness of the need for more deliberate focus on translation and implementation in the obesity prevention field is motivated by an interest in improving intervention outcomes related to prevention of excess weight gain or achieving initial or sustained weight control. Findings of "state-of the-art" obesity interventions in some federally funded consortia studies are driving efforts to deconstruct intervention approaches in the realm of behavior change - to "unpack the black box" and develop deeper understandings. ${ }^{16,17}$ Tate et al. describe this as "a new frontier" in the effort to improve complex multicomponent obesity interventions, allowing for estimating dose and pointing to aspects that may need more or less emphasis under various conditions. ${ }^{17}$ The Tate et al. review may be especially informative for those of us who are new to this use of taxonomic approaches. These authors also comment on other relevant taxonomies or classifica- tion systems, discuss challenges inherent in taxonomic approaches, and make recommendations for advancing this line of endeavor.

Because health disparities and health equity-oriented research emphasize understanding of and adapting to context, implementation research is viewed as especially relevant to such research in both health care delivery and community settings. ${ }^{18,19}$ The contexts to be addressed through research translation differ from those in controlled research settings. In disparities research they differ in specific ways related to social or economic circumstances or policies that adversely influence the health of marginalized populations. If not addressed, these influences undercut effectiveness by limiting the feasibility of adherence or posing deterrents to adoption of recommended behaviors. ${ }^{8}$ Insufficient attention to contexts is thought to explain findings of ineffectiveness or lesser effectiveness of obesity prevention interventions in racial/ethnic minority or lower income communities compared with predominantly white or higher income communities. As described elsewhere, the Healthy Communities Study, a nationwide observational study of body mass index changes associated with delivery of recommended interventions, found that the favorable results were observed in the northeast but not in other regions and were only observed in higher income white communities and not shared by lower income, African American, or Hispanic children or communities. ${ }^{20}$ This might have resulted from differential community capacity to implement the interventions or differential uptake due to contextual factors. A taxonomic approach such as that used for COEB would facilitate identification and analysis of contextual variables through 
a variety of qualitative or quantitative approaches. Taxonomies also reveal gaps in study reporting, for example, when potentially relevant contextual variables are not reported or identifiable through other means.

\section{Complementary Resources}

A scan for other taxonomies identified some approaches that might be relevant to obesity interventions and useful in conjunction with the COEB database as it currently stands or as expanded to other age groups or intervention types. One that relates directly to childhood obesity interventions used a taxonomic approach to develop a nine-component typology (a broad conceptual classification) of community intervention approaches - both macrolevel (e.g., policy and environmental change) and microlevel (directly targeting changes in individual behavior) - based on studies conducted in Los Angeles County in 2000-2016. ${ }^{21}$ This typology was used to develop a quantitative Community Intervention Dose Index by coding each strategy for strength (expert judgments of effectiveness), estimated percentage of the target population reached (information from program grantees or, if necessary, expert judgment), and fidelity (evidence as to whether the intervention was implemented as intended). ${ }^{22}$ Typology of Interventions in Proximal Physical Micro-Environments focuses on classifying strategies based on behavioral economics theory ("nudge" approaches, or interventions on "choice architecture"). ${ }^{23}$ This classification identifies behavior change interventions intended to work through changing placement or properties of products, objects associated with the products, or the wider retail or marketing environment.

Two health equity-related taxonomic resources are also noteworthy, both developed with processes like that used by COEB. One was based on a scoping review of disparities research conducted in health care settings. This taxonomy categorizes identified research components in terms of strategies and tactics used to change behavior, systems, or services, and the levels at which the intervention was targeted (e.g., providers, patients, system, organization, community, or policy). ${ }^{24}$ The other resource is a tool kit consisting of a typology of cultural adaptation approaches for behavior change interventions, by intervention stages and contexts, with a decision-making tool to guide its use (Relevance, Evidence base, Stages of Intervention, Ethnicity, and Trends). ${ }^{25}$ Most studies identified in the scoping review on which the taxonomy was based were US studies that focused on African American populations and on healthy eating or physical activity. Cultural tailoring was identified as an intervention component in a substantial proportion of studies in the COEB pilot. ${ }^{10}$ However, although the rationale for such tailoring is well established, its role in intervention efficacy and effectiveness is unclear. The COEB database offers the potential for systematic consideration and hypothesis development about the role of cultural tailoring.

\section{Next Steps}

The limitations of conventional meta-analysis approaches for identifying solutions to obesity and other complex health problems are well known. ${ }^{26}$ Conventional methods are not informative about why interventions are or are not effective and are not designed to consider external validity. Taxonomic approaches can support implementation research to answer questions about what it takes for an intervention to be well designed and delivered for effectiveness in diverse settings, including contexts related to health equity. Expansion of the COEB database to include studies from other high-income countries would be an important next step, to increase the number of studies for consideration and take advantage of the greater variability in economic, policy, and community contexts, and learn from successes. In the short term, and to support the case for devoting more resources to this type of implementation research, it would be important to make full use of the COEB database to probe for novel insights and develop new hypotheses about ways to improve childhood obesity prevalence and trajectories and address disparities.

\section{References}

1. National Academies of Science Engineering and Medicine. Driving Action and Progress on Obesity Prevention and Treatment. Proceedings of a Workshop. The National Academies Press: Washington, DC, 2017.

2. Institute of Medicine. Accelerating Progress in Obesity Prevention: Solving the Weight of the Nation. The National Academies Press, Washington, DC, 2012.

3. Chaparro MP, Anderson CE, Crespi CM, et al. The new child food package is associated with reduced obesity risk among formula fed infants participating in the Special Supplemental Nutrition Program for Women, Infants and Children (WIC) in Los Angeles County, California, 2003-2016. Int J Behav Nutr Phys Act 2020; 17:18.

4. Pan L, Blanck HM, Park S, et al. State-specific prevalence of obesity among children aged 2-4 years enrolled in the special supplemental nutrition program for women, infants, and children-United States, 2010-2016. MMWR Morb Mortal Wkly Rep 2019;68:1057-1061.

5. National Center for Health Statistics. Health, United States, 2018 National Center for Health Statistics: Hyattsville, MD, 2019.

6. Ogden CL, Fryar CD, Hales CM, et al. Differences in obesity prevalence by demographics and urbanization in US children and adolescents, 2013-2016. JAMA 2018;319:2410-2418.

7. Dietz WH. We need a new approach to prevent obesity in lowincome minority populations. Pediatrics 2019;143:e20190839.

8. Kumanyika SK. A framework for increasing equity impact in obesity prevention. Am J Public Health 2019;109:1350-1357.

9. Kumanyika S. Overcoming inequities in obesity: What don't we know that we need to know? Health Educ Behav 2019;46:721727.

10. King H, Magnus M, Hedges LV, et al. Childhood Obesity Evidence Base Project: Methods for taxonomy development for application in taxonomic meta-analysis. Childhood Obes 2020;16: S2-7-S2-20. 
11. Hedges LV, Saul JD, Cyr C, et al. Childhood Obesity Evidence Base Project: A rationale for taxonomic versus conventional metaanalysis. Childhood Obes 2020;16:S2-1-S2-6.

12. Young-Hyman D, Kettel Kahn L. Childhood Obesity Evidence Base Project. Building translational capacity through meta-analytic methods. Childhood Obes 2020;16:S2-49-S2-54.

13. Scott-Sheldon LAJ, Hedges LV, Cyr C, et al. Childhood Obesity Evidence Base Project. A systematic review and meta-analysis piloting a new taxonomy of intervention components to improve weight status in children 2-5 years of age, 2005-2019. Childhood Obes 2020;16:S2-21-S2-48.

14. Economos CD, Haire-Joshu D. Preventing obesity in 2-5 year olds: A pathway to advancing intervention research. Childhood Obes 2020;16:S2-59-S2-61.

15. McLeroy KR, Bibeau D, Steckler A, Glanz K. An ecological perspective on health promotion programs. Health Educ $Q 1988$; 15:351-377.

16. Belle SH, Stevens J, Cella D, et al. Overview of the obesity intervention taxonomy and pooled analysis working group. Transl Behav Med 2016;6:244-259.

17. Tate DF, Lytle LA, Sherwood NE, et al. Deconstructing interventions: Approaches to studying behavior change techniques across obesity interventions. Transl Behav Med 2016;6:236-243.

18. Chin MH, Goldmann D. Meaningful disparities reduction through research and translation programs. JAMA 2011;305:404-405.

19. Mazzucca S, Arredondo EM, Hoelscher DM, et al. Expanding implementation research to prevent chronic diseases in community settings. Annu Rev Public Health (in press).

20. Kumanyika SK. Supplement overview: What the healthy communities study is telling us about childhood obesity prevention in U.S. communities. Pediatr Obes 2018;13 Suppl 1:3-6.
21. Robles B, Jiang LH, Prelip M, et al. Notes from the field: Creating a typology of childhood obesity intervention strategies. Eval Health Prof 2020;43:131-134.

22. Wang MC, Crespi CM, Jiang LH, et al. Developing an index of dose of exposure to early childhood obesity community interventions. Prev Med 2018;111:135-141.

23. Hollands GJ, Bignardi G, Johnston M, et al. The TIPPME intervention typology for changing environments to change behaviour. Nat Hum Behav 2017;1:0140.

24. Clarke AR, Goddu AP, Nocon RS, et al. Thirty years of disparities intervention research: What are we doing to close racial and ethnic gaps in health care? Med Care 2013;51:1020-1026.

25. Davidson EM, Liu JJ, Bhopal RAJ, et al. Behavior change interventions to improve the health of racial and ethnic minority populations: A tool kit of adaptation approaches. Milbank Quarterly 2013;91:811-851.

26. Institute of Medicine. Bridging the Evidence Gap in Obesity Prevention: A Framework to Inform Decision Making. The National Academies Press: Washington, DC, 2010.

Address correspondence to:

Shiriki K. Kumanyika, PhD, MPH

Department of Community Health and Prevention

Drexel University Dornsife School of Public Health

3215 Market Street, 4th Floor

Philadelphia, PA 19104

USA

E-mail: kumanyika@drexel.edu 